\title{
Structural-functional correlations between hippocampal volume and cortico-limbic emotional responses in depressed children
}

\author{
Hideo Suzuki • Kelly N. Botteron • Joan L. Luby • \\ Andy C. Belden - Michael S. Gaffrey • Casey M. Babb • \\ Tomoyuki Nishino • Michael I. Miller • \\ J. Tilak Ratnanather • Deanna M. Barch \\ Published online: 5 October 2012 \\ (C) Psychonomic Society, Inc. 2012
}

\begin{abstract}
Although hippocampal atrophy and altered functional brain responses to emotional stimuli have been found in major depressive disorder (MDD), the relationship between the two is not yet well understood. The present study focused on children with and without a history of preschool onset MDD (PO-MDD) and directly examined the relations between hippocampal volume and functional brain activation to affect-eliciting stimuli. Children completed annual diagnostic assessments starting at preschool. When children were school-aged, high-resolution structural MRI and taskrelated functional MRI data were acquired from $N=64$ nonmedicated children. During fMRI, subjects were shown emotional faces. Results from the total sample indicated that smaller bilateral hippocampal volumes were associated with greater cortico-limbic (e.g., amygdala, hippocampus, dorsolateral prefrontal cortex) activation to sad or negative faces
\end{abstract}

H. Suzuki $(\triangle) \cdot$ K. N. Botteron · J. L. Luby • A. C. Belden •

M. S. Gaffrey $\cdot$ C. M. Babb • T. Nishino $\cdot$ D. M. Barch

Department of Psychiatry,

Washington University School of Medicine,

Campus Box 8134, 660 Euclid Ave.,

Saint Louis, MO 63110, USA

e-mail: suzukih@psychiatry.wustl.edu

K. N. Botteron • D. M. Barch

Department of Radiology, The Edward Mallinckrodt Institute of Radiology, Washington University School of Medicine,

Saint Louis, MO, USA

M. I. Miller $\cdot$ J. T. Ratnanather

Center for Imaging Science, Johns Hopkins University,

Baltimore, MD, USA

M. I. Miller $\cdot$ J. T. Ratnanather

Department of Biomedical Engineering,

The Whitaker Biomedical Engineering Institute,

Johns Hopkins University,

Baltimore, MD, USA versus neutral faces. Left hippocampal volume was negatively associated with the cortico-limbic activation in both the PO-MDD and healthy children. Right hippocampal volume was negatively correlated with amygdala responses in the PO-MDD group, but not in the healthy comparison group. These findings suggest that there may be important interrelationships between reduced hippocampal volume and hyperactivation of brain responses in children, both those with and those without a history of PO-MDD.

Keywords Hippocampus · sMRI · fMRI $\cdot$ Child $\cdot$ Preschool depression

The neurobiological mechanisms contributing to major depressive disorder (MDD) have been increasingly investigated

M. I. Miller $\cdot$ J. T. Ratnanather

The Institute for Computational Medicine,

Johns Hopkins University,

Baltimore, MD, USA

D. M. Barch

Department of Psychiatry, The Silvio Conte Center

for Neuroscience of Mental Disorders,

Washington University School of Medicine,

Saint Louis, MO, USA

D. M. Barch

Department of Psychology, Washington University in St. Louis, Saint Louis, MO, USA 
to advance our understanding of the etiology, diagnosis, and treatment of this illness (Drevets, Price, \& Furey, 2008; Savitz \& Drevets, 2009). In particular, structural MRI (sMRI) and functional MRI (fMRI) have been used to identify alterations in both the volume and shape of brain regions in MDD, as well as differences in functional brain activation during a variety of emotion-processing and emotion-regulation paradigms. For example, sMRI studies have frequently reported reduced hippocampal volume in MDD (Lorenzetti, Allen, Fornito, \& Yucel, 2009; McKinnon, Yucel, Nazarov, \& MacQueen, 2009; Sapolsky, 2000a; Savitz \& Drevets, 2009). Such reductions are found in elderly (O'Brien, Lloyd, McKeith, Gholkar, \& Ferrier, 2004; Steffens et al., 2000; Steffens, McQuoid, Payne, \& Potter, 2011) and middle-aged MDD populations (Bremner et al., 2000; Frodl et al., 2002; Malykhin, Carter, Seres, \& Coupland, 2010), even when controlling for total brain/intracranial volume. However, results in younger populations (between the ages of 8 and 21 years) have been less consistent than those for depressed adults; some studies have shown that hippocampal volume is reduced in depressed adolescents and children after controlling for total brain/intracranial volume (Caetano et al., 2007; MacMaster \& Kusumakar, 2004; MacMaster et al., 2008; Rao et al., 2010), whereas other studies did not find such hippocampal differences (MacMillan et al., 2003; Rosso et al., 2005). Such mixed findings in depressed children or adolescents may suggest that hippocampal volume reductions reflect the impact of risk factors, such as early adversity/trauma or increased stress responsivity, that may take some time to accumulate; thus, differences may be more robust and detectable in adult depression.

Alternatively, the conflicting data for hippocampal reduction in pediatric MDD may be partially explained by evidence for variability in the severity of reduced hippocampal volume across studies and across individuals. For example, reduced absolute/normalized volume of the hippocampus is greater in MDD patients with longer episode durations (Bell-McGinty et al., 2002; Colla et al., 2007; MacQueen et al., 2003; McKinnon et al., 2009; Sheline, Sanghavi, Mintun, \& Gado, 1999; Sheline, Wang, Gado, Csernansky, \& Vannier, 1996), more/severe symptoms (Colla et al., 2007; MacQueen et al., 2003; McKinnon et al., 2009; Vakili et al., 2000), early-life adversity (Rao et al., 2010), poorer response to antidepressant treatment (Sheline, Gado, \& Kraemer, 2003; Vakili et al., 2000), or more severe MDDrelated cognitive dysfunction (Frodl et al., 2006). Such results raise the important question of whether individual differences in volume in the hippocampus are also associated with other neurobiological characteristics of MDD, such as alterations in functional brain responses to affect-eliciting stimuli.

A number of fMRI studies have shown alterations in functional brain responses during negative emotion-processing paradigms in MDD (or in individuals at high risk for MDD), including increased activation of limbic-paralimbic regions (e.g., amygdala, putamen, thalamus) (Fales et al., 2009, 2008; Fu et al., 2004; Monk et al., 2008; Roberson-Nay et al., 2006; Savitz \& Drevets, 2009; Sheline et al., 2001, 2009; Siegle, Steinhauer, Thase, Stenger, \& Carter, 2002; Surguladze et al., 2005) and altered activation of prefrontal regions (PFC; e.g., dorsolateral PFC) (Fales et al., 2009; Hamilton et al., 2012; Siegle, Thompson, Carter, Steinhauer, $\&$ Thase, 2007) thought to be involved in emotion regulation (Maletic et al., 2007; Phillips, Drevets, Rauch, \& Lane, 2003). Interestingly, MDD is also associated with increased activation to negative stimuli in the hippocampus itself (Sheline et al., 2009). Studies with healthy individuals have further suggested differential functions of hippocampal subregions: activation of the anterior hippocampus is usually observed in response to emotionally charged stimuli, whereas the posterior hippocampus seems to be activated to encoding of emotionally neutral stimuli (Dolcos, LaBar, \& Cabeza, 2004; Small, Schobel, Buxton, Witter, \& Barnes, 2011). However, to date, no research has directly examined the potential relationships between individual variability in hippocampal volume and variability in functional brain activation in either healthy or MDD populations.

There are at least two reasons to hypothesize such relationships. The first is that the integrity of the hippocampus may influence responses to emotional stimuli. For example, preclinical studies have shown that hippocampal lesions lead to affective disturbances, including impaired fear acquisition (Anagnostaras, Maren, \& Fanselow, 1999; Ji \& Maren, 2007; Otto \& Poon, 2006; Yoon, Graham, \& Kim, 2011), reduced affiliation, emotional overreactivity, and increased aggression (Machado \& Bachevalier, 2006). Although hippocampal lesions are not equivalent to reduced hippocampal volume, these findings are consistent with the possibility that reduced hippocampal volume may contribute to altered emotional processing and, potentially, to the development of MDD. It has also been suggested that there are some brain regions where functional response to emotional stimuli may be modulated by the hippocampus. The anterior hippocampus projects to the amygdala and medial PFC, whereas the posterior hippocampus projects to the posterior cingulate cortex (Small et al., 2011). The literature on MDD postulates that the hippocampus also connects with the basal ganglia (Botteron, 2001; Drevets et al., 2008; Price \& Drevets, 2010). Therefore, reduced hippocampal volume may be associated with impaired function of cortico-limbic emotion regions (e.g., amygdala, medial PFC, cingulate cortex, and basal ganglia) with neural projections from the hippocampus. This could manifest as altered emotional behavior and differences in brain activation to emotioneliciting stimuli. 
A second reason to contemplate a possible association between hippocampal volume and affect-elicited brain activation is that the degree of functional responsiveness to emotionally evocative stimuli (e.g., stress, trauma, lifethreatening situations) could impact hippocampal volume. For example, increased stress/trauma exposure is associated with reduced hippocampal volume in both preclinical (Blugeot et al., 2011) and clinical (Karl et al., 2006; Woon, Sood, \& Hedges, 2010) studies, probably due to excitotoxic effects of excessive glucocorticoids on hippocampal neurons (McEwen, 2000; McEwen, Eiland, Hunter, \& Miller, 2011; Sapolsky, 2000a, b). In MDD, emotional responsivity has been studied both in terms of increased hypothalamic-pituitary-adrenal (HPA) axis stress reactivity (Burke, Davis, Otte, \& Mohr, 2005; Lopez-Duran, Kovacs, \& George, 2009; Luby, Heffelfinger, et al., 2003) and in terms of functional brain responses to emotional stimuli, and studies in adults (Fales et al., 2008; Fu et al., 2004; Sheline et al., 2001, 2009; Siegle et al., 2002; Surguladze et al., 2005), adolescents (Roberson-Nay et al., 2006), and children (Barch, Gaffrey, Botteron, Belden, \& Luby, in press; Gaffrey, Luby et al., 2011) with MDD have shown increased amygdala responses to emotional stimuli or emotionally evocative stimuli. Although increased functional responses to emotional stimuli are not equivalent to experiencing trauma or life threatening situations, these findings do suggest a pattern of heightened emotional responsivity to negative stimuli in MDD. Such altered emotional responsivity in the amygdala may possibly impact hippocampal structure because the amygdala has neural projections to the hippocampus, especially its anterior region (Small et al., 2011) and because the hippocampus is clearly sensitive to stressrelated excitotoxicity. Moreover, sMRI studies suggest that hippocampal volume reduction in MDD is associated with duration of illness (Bell-McGinty et al., 2002; Colla et al., 2007; MacQueen et al., 2003; McKinnon et al., 2009; Sheline et al., 1999, 1996), which has been hypothesized to reflect hippocampal volume reduction resulting from the accumulation of stressors (McKinnon et al., 2009; Sheline et al., 1999). Although children have had less time to accumulate "stressors," as compared with adults, it is nonetheless possible that increased responsivity to emotionally evocative stimuli could be associated with reduced hippocampal volume even at a relatively younger age (perhaps due to greater neuroplasticity).

Therefore, the first goal of the present study was to examine the relationships between hippocampal volume and functional brain responses to affect-eliciting stimuli. The second goal was to further examine the neurobiological changes present in very early onset MDD. The majority of MRI research has focused on depressed adolescents and adults (i.e., 9 years of age or older). However, MDD has been also described in children as young as preschool age (i.e., ages between 3 and 6), referred to as preschool onset MDD (PO-MDD) (Luby, Si, Belden, Tandon, \& Spitznagel, 2009). PO-MDD is a validated clinical syndrome, characterized by DSM-IV-defined depressive symptoms during preschool age (Luby, Belden, Sullivan, et al., 2009; Luby, Mrakotsky, et al., 2003; Luby et al., 2006). Despite potential differences in the duration of accumulation of stress or a history of depressive episodes between PO-MDD and adulthood MDD, there is emerging evidence that neurobiological alterations associated with PO-MDD are similar to those seen in depressed adolescents and adults. For example, in our prior work, the severity of depression in preschool-aged children with current PO-MDD, as well as school-aged children with a history of PO-MDD, was associated with increased activation of regions in the limbic system, such as the amygdala and hippocampus, in response to sad faces relative to neutral faces (Barch et al., in press; Gaffrey et al. 2011b; Luking et al., 2011). We hypothesized that reduced hippocampal volume would be correlated with altered functional cortico-limbic responses to negative stimuli among children. The present study focused on functional activation in specific cortico-limbic regions, selected on the basis of the prior fMRI studies on PO-MDD and MDD discussed above: the amygdala, hippocampus, basal ganglia, medial and dorsolateral PFC, and anterior cingulate cortex. We hypothesized that smaller hippocampal volumes would be negatively correlated with functional activation to negative faces in emotional processing/limbic regions such as the amygdala, hippocampus, basal ganglia, and potentially the anterior cingulate, but they would be positively correlated to functional activation in emotion-regulation regions in the PFC. In addition, we hypothesized that these relationships would be present in both healthy children and children with PO-MDD, although they might be stronger in PO-MDD children if there is more variability in their hippocampal volumes or functional activation responses as a function of their MDD status.

\section{Method}

\section{Subjects}

Between the years of 2003 and 2005, preschoolers between the ages of 3.0 and 5.11 were recruited from pediatricians' offices, daycares, and preschools in the St. Louis metropolitan area, using a screening checklist to oversample preschoolers with symptoms of MDD into the Validation of Preschool Depression Study (PDS), a longitudinal study of PO-MDD. Written consent was obtained from parents, and all study procedures were approved in advance by the Institutional Review Board. A sample of 306 children, without exclusion criteria (head trauma, neurological disease, or severe 
developmental delays or prematurity), were assessed at baseline, and 257 of them completed two follow-up annual waves, using an age-appropriate diagnostic interview, the Preschool Age Psychiatric Assessment (PAPA; Egger, 2009; Egger, Ascher, \& Angold, 1999/2003; Egger et al., 2006). On the basis of the PAPA diagnoses, children were classified into those with a history of PO-MDD, with other psychiatric disorders without PO-MDD, or with healthy status.

At the time of this report, usable data were available from 85 children who participated in structural and functional imaging 4-8 years after the baseline assessment, when they were between the ages of 7 and 12 . There were additional 25 children who underwent scanning but who did not provide usable data. Five of these children could not complete the scanning proto$\mathrm{col}$, and 20 had poor quality data (average signal-to-noise ratio [SNR] less than 150 on both face task runs). We focused on SNR for exclusion rather than movement alone, since this tends to be a more conservative metric that captures both movement effects and scan quality. This is a loss rate of approximately $20 \%$, which is typical for developmental neuroimaging studies. Twenty one of the 85 children with usable data were excluded because they either were currently taking psychotropic medicines or had taken them in the past. Therefore, a total of $N=64$ nonmedicated children were included in the current analysis: $n=24$ healthy children (i.e., no psychiatric diagnosis across all waves), $n=27$ children with a history of PO-MDD (i.e., MDD at least once before age of 6 across waves), and $n=13$ children with a history of other diagnoses without PO-MDD (i.e., disruptive or anxiety disorders at least once but no MDD diagnosis across waves). Our final sample was representative of children who were free from potential effects of psychotropic medication.

After processing structural imaging data, the volumetric data of the right hippocampus from 2 healthy subjects and the left hippocampal data from 10 subjects ( 3 healthy, 5 POMDD, and 2 other diagnosis) did not pass quality control for segmentation of the hippocampus (see below for details). Thus, the present study contained 62 subjects ( 22 healthy, 27 MDD, 13 other) in all analyses of right hippocampal volume and 54 subjects ( 21 healthy, $22 \mathrm{MDD}, 11$ other) in all analyses of left hippocampal volume.

Mock scanner training for children

After obtaining consent, children participated in a training sequence designed to increase their comfort and understanding of the scanning procedure and to minimize in-scan movement. Training included watching a short video following another child through their scanning experience, followed by the use of a mock scanner (i.e., MRI simulator) to introduce the scanning environment. Children were allowed to complete this experience at their own pace, and each training session was altered to meet the needs of the individual subject.
Generally, this included lying down on the scanner table, having headphones placed on their head, and entering the bore of the simulator. Once inside the bore, children still practiced lying while listening to recorded MRI sounds and receiving visual feedback of their head movement in real time (MoTrak software). After approximately $10 \mathrm{~min}$ of practice, children were removed from the simulator, and any remaining questions regarding the practice or actual scan were addressed.

\section{Diagnostic assessment}

Trained staff from the Early Emotional Development Program (EEDP) at Washington University School of Medicine conducted up to three in-person annual interview sessions using the PAPA with child subjects and their caregivers. Approximately $20 \%$ of the interviews were audiotaped and reviewed for reliability and quality control as previously reported (Luby, Belden, Pautsch, Si, \& Spitznagel, 2009). The PAPA consists of a series of developmentally appropriate questions answered by the primary caregiver, which cover the DSM-IV criteria for Axis I disorders that may arise in preschoolers, including MDD, posttraumatic stress disorder (PTSD), general anxiety disorder (GAD), separation anxiety disorder (SAD), oppositional defiant disorder (ODD), attention deficit/hyperactivity disorder (ADHD), and conduct disorder (CD). The 2-week duration of symptoms requirement for MDD during preschool was set aside on the basis of data suggesting that it may not be an appropriate threshold for this developmental period (Gaffrey, Belden, \& Luby, 2011; Luby et al., 2002, 2009c). Otherwise, preschoolers who fell into the MDD group met all DSM-IV MDD symptom criteria.

Children were classified as having a history of PO-MDD if they displayed clinical levels of MDD on the PAPA at any time point prior to the age of 6.0 (Luby et al., 2002). The PAPA also allows for quantification of symptom severity: for each inperson diagnostic time point, depression sum scores (the total number of MDD symptoms endorsed in the PAPA) were calculated and used to assess core MDD symptom severity (Luby, Mrakotsky, Heffelfinger, Brown, \& Spitznagel, 2004). We also assessed symptoms of non-MDD internalizing disorders (PTSD, GAD, and SAD) and externalizing disorders (ODD, ADHD, and CD) at each time point. We used these diagnostic characteristics to examine whether results were specific to PO-MDD or were related more generally to childhood internalizing or externalizing psychopathology.

Functional task and stimuli

The fMRI task was a facial emotion-processing task, using the MacArthur Network Face Stimuli Set, a validated stimulus set that contains 43 different actors from different ethnic/cultural 
backgrounds (Tottenham et al., 2009). Children were shown faces that varied in affective content (sad, angry, fearful, happy, and neutral) from 10 sets of adults and were asked to decide whether the face was male or female. We chose to use a task that did not require explicit attention to the emotional content, because of evidence that heightened amygdala responses associated with MDD may be more apparent with tasks that do not explicitly require a focus on the emotional content of the stimuli (Fales et al., 2008; Monk et al., 2008). In addition, we created intermediate affective expressions by morphing the neutral expression for each individual with their emotional expression so that the resulting face was halfway between neutral and the target emotion (MorphAge software). We included these stimuli because behavioral and brain activation biases in depression may be more apparent at less intense emotional expressions where there may be a greater likelihood of detecting bias. Thus, each "actor" in the stimulus set provided a total of nine facial expressions (neutral; $50 \%$ sad, angry, fearful, and happy; $100 \%$ sad, angry, fearful, and happy). Each stimulus was presented for 2,500 ms, followed by an ITI ranging between 500 and $6,500 \mathrm{~ms}$. The task was programmed in PsyScope, and behavioral responses in the scanner were acquired via a fiber optic button box interfaced with the PsyScope button box. Each run consisted of 45 stimuli, five from each of the nine conditions. Selection of the specific face to be presented on each trial was determined by PsyScope using a random without replacement algorithm, within the constraint of the number of faces of each type to be presented in a run. The images were projected onto a computer screen behind the subject's head within the imaging chamber. The screen was viewed by a mirror positioned approximately $8 \mathrm{~cm}$ above the subject's face.

\section{Data acquisition}

Structural and functional scanning was performed on a 3.0 Tesla TIM TRIO Siemens whole-body system within the same session. Each session took approximately $1 \mathrm{~h} 15 \mathrm{~min}$. Two three-dimensional T1-weighted magnetization prepared rapid gradient echo (MPRAGE) scans ( $\sim 6 \mathrm{~min}$ each; $\mathrm{TR}=2,400 \mathrm{~ms}, \mathrm{TE}=3.16 \mathrm{~ms}$, flip angle $=8^{\circ}$, slab $=$ $160 \mathrm{~mm}, 160$ slices, isotropic $1 \times 1 \times 1 \mathrm{~mm}$ voxels on a matrix of $256 \times 224 \times 160$, 1 signal average) were acquired in the sagittal plane. Blood oxygen level dependent (BOLD) images during face processing were acquired with a $\mathrm{T} 2 *$ weighted asymmetric spin-echo echo-planar sequence $\left(\sim 5 \mathrm{~min} ; \mathrm{TR}=2,500 \mathrm{~ms}, \mathrm{TE}=27 \mathrm{~ms}\right.$, flip angle $=90^{\circ}$, FOV $=256 \mathrm{~mm}$, voxel size $=4 \times 4 \times 4 \mathrm{~mm}$ ) in the axial plane parallel to the anterior-posterior commissure, with a 12-channel head coil. During each functional run, 99 sets of 36 contiguous axial images with isotropic voxels $\left(4 \mathrm{~mm}^{3}\right)$ were acquired parallel to the anterior-posterior commissure plane. These functional face processing scans took approximately $12 \mathrm{~min}$. In addition, the children completed a film-processing task $(\sim 5 \mathrm{~min})$, two resting state runs $(\sim 12 \mathrm{~min})$, and two diffusion tensor imaging runs $(\sim 15 \mathrm{~min})$.

\section{Structural data processing}

The 16-bit MPRAGE imaging data were converted to Analyze format (Robb, 2001). The two acquisitions were then coregistered and summed to increase the SNR (Holmes et al., 1998). The registered images were resampled to $0.5 \mathrm{~mm}^{3}$ voxels and then converted to 8 -bit while maintaining the dynamic range of the image intensities.

The target hippocampus was rotated to its head/tail orientation and was divided into five coronal parts. Then, four landmarks (superior, lateral, inferior, and medial) were placed on these coronal views of the hippocampus (Csernansky et al., 1998) by an experienced rater (C.M.B.) who was blind about diagnostic classification. The same procedure was applied to place landmarks on the template hippocampus, which an experienced rater (C.M.B.) manually delineated on an anatomically representative individual drawn from the same age population on the basis of our previously described hippocampal boundary definitions (Haller et al., 1996, 1997). The template hippocampus was then converted to a 3-D tessellated surface to generate the template surface.

The landmarked subject hippocampus was oriented to the template hippocampus by applying a rigid landmark transformation algorithm (no stretch or warping). A nonlinear large deformation landmark (LDL) transformation was then applied to refine the initial transformation. These two transformations aligned the subject hippocampus with the template hippocampus purely on the basis of expert landmarks. A nonlinear diffeomorphic transformation was then generated using the voxel intensities of the subject and template image through the large deformation diffeomorphic metric mapping (LDDMM; Beg, Miller, Trouve, \& Younes, 2005; Miller, Trouve, \& Younes, 2002). The inverse of the highdimensional transformation from LDDMM was applied to the template surface. Finally, the LDL transformation was inverted to generate a surface representation of the subject hippocampus. Hippocampal volumes were calculated within the resulting surfaces.

Although our transformation technique performs with high accuracy rates, there are generally a small proportion of subject scans whose final transformation results do not meet quality check (QC) standards assessed during visual review of all hippocampi. Visual manifestations of QC problems include surface boundaries that extend into the cerebral spinal fluid and outside of the hippocampal gray matter. Two right hippocampi and ten left hippocampi did not meet our QC standards and were excluded in subsequent analyses. 
Functional data preprocessing

The fMRI data were preprocessed using the following steps: (1) compensation for slice-dependent time shifts; (2) removal of the first four images of each run to allow BOLD signal to reach steady state; (3) elimination of odd/even slice intensity differences due to interpolated acquisition; (4) realignment of data acquired in each subject within and across runs to compensate for rigid body motion (Ojemann et al., 1997); (5) intensity normalization to a whole brain mode value of 1,000 ; (6) registration of the 3-D structural volume (T1) to the atlas representative template in the Talairach coordinate system (Talairach \& Tournoux, 1988) using a 12-parameter affine transform and resampling to 1$\mathrm{mm}$ cubic representation (Buckner et al., 2004; Ojemann et al., 1997); (7) coregistration of the 3-D fMRI volume to the $\mathrm{T} 2$, and the T2 to the subjects' structural image; (8) transformation of the fMRI to atlas space using a single affine 12-parameter transform that included resampling to a 3-mm cubic representation; and (9) spatial smoothing using a 6$\mathrm{mm}$ full-width half-maximum Gaussian filter. Prior research has validated the use of this approach with children in our age range (Burgund et al., 2002; Kang, Burgund, Lugar, Petersen, \& Schlaggar, 2003). This validation has included evidence that, when a child-specific atlas is used (as we did), the variation in the degree of volumetric displacement in children 7 years of age and older following registration to a standard space is no more than that for adults and that children (7 years and older) and adults show similar hemodynamic response functions to basic visual and motor processing.

Analysis of fMRI data was performed using in-house software (FIDL analysis package, http://www.nil.wustl.edu/labs/fidl/index.html) utilized in numerous previously published studies (Becerril, Repovs, \& Barch, 2011; Braver, Paxton, Locke, \& Barch, 2009; Gaffrey et al. 2011b; Jimura, Locke, \& Braver, 2010; Ollinger, Corbetta, \& Shulman, 2001; Ollinger, Shulman, \& Corbetta, 2001; Savine \& Braver, 2010). Estimates of BOLD response to each face type within each subject were obtained using fixed effects general linear models incorporating regressors for linear trend and baseline shifts. A hemodynamic response shape was assumed (Boynton function) and used to derive magnitude estimates relative to fixation baseline. These singlesubject estimates were then entered into group-level analyses that treated subjects as random effects.

\section{Analytical approach}

Demographic and clinical differences (age, gender, handedness, PO-MDD diagnosis, average core MDD severity over the three annual assessments, and comorbid non-MDD internalizing/externalizing diagnosis) between the PO-MDD and healthy groups were tested using independent-samples $t$-tests or cross tabulation chi-square tests. Moreover, additional independent-samples $t$-tests were used to compare right and left hippocampal volumes across groups.

The present study used a priori region of interest (ROI) analysis for the correlations between hippocampal volume and functional brain responses to negative stimuli. The selected ROIs were those previously thought to be relevant for understanding emotion processing in MDD: the amygdala, hippocampus, basal ganglia, medial and dorsolateral PFC, and anterior cingulate cortex. Previous fMRI studies on PO-MDD or MDD have identified functional alterations in the amygdala (Barch et al., in press; Fales et al., 2008, 2009; Fu et al., 2004; Gaffrey et al. 2011b; Roberson-Nay et al., 2006; Sheline et al., 2001, 2009; Siegle et al., 2007; Surguladze et al., 2005), dorsolateral PFC (Fales et al., 2009), hippocampus (Barch et al., in press; Sheline et al., 2009), medial PFC (Sheline et al., 2009), anterior cingulate cortex (Fu et al., 2004; Sheline et al., 2009), and basal ganglia (Fu et al., 2004), while it has also been postulated that the hippocampus structurally interacts with the amygdala, medial PFC, cingulate cortex, and basal ganglia (Botteron, 2001; Drevets et al., 2008; Price \& Drevets, 2010; Small et al., 2011) within emotional processing network. Our a priori selection of ROIs was based on these extant literatures.

Our anatomical templates of the amygdala, hippocampus, and basal ganglia were derived from prior work (Mamah et al., 2007, 2010; Wang et al., 2008). The PFC ROIs were defined on an atlas-representative image using the boundaries described by Rajkowska and Goldman-Rakic (1995a, b). The cingulate ROIs were used from centroids of activation identified in prior studies (Fales et al., 2008, 2009; Sheline et al., 2009), around which we drew 25-mm diameter spherical ROIs edited to respect gray matter boundaries on an atlas-representative image.

We examined voxel-based analysis of partial correlations (controlling for age and sex) between left/right hippocampal volume and two indices of functional brain responses to emotional faces: (1) activation to $50 \%$ and $100 \%$ sad faces, relative to neutral faces, and (2) activation to the average of all negative face types (50\% and $100 \%$ sad, angry, and fearful faces), relative to neutral faces. These tests were performed both for the entire nonmedicated sample and just within each of the PO-MDD or healthy groups, with correction for multiple comparisons using simulations to generate a $p$-value and cluster size criterion that provide a false positive rate of $p<.05$ for the whole ROI mask (Forman et al., 1995; McAvoy, Ollinger, \& Buckner, 2001). This threshold/cluster-size requirement provides protection against type I error and was chosen on the basis of Monte Carlo simulations via AlphaSim (Ward, 2000). The present study set the threshold as $z=2.6$ at $p<.005$ and 16 voxels. If any significant correlations were found, we tested whether they remained significant after controlling for a cumulative 
history of PO-MDD status, non-MDD internalizing disorders, and/or externalizing disorders. This was measured by assessing whether or not subjects had ever met criteria for PO-MDD, a non-MDD internalizing disorder, and/or an externalizing disorder between the baseline assessment and the follow-up assessments prior to the present MRI study. In addition, we investigated whether the correlations found in the entire sample remained significant (or similar in magnitude) within the healthy control or PO-MDD group alone.

To examine any group difference in the correlations, we used hierarchical regression analyses to predict the magnitude of BOLD in the regions identified in the initial correlational analyses, with age, sex, PO-MDD status, and hippocampal volume entered at step 1 and the cross-product of PO-MDD status and hippocampal volume at step 2 .

\section{Results}

Demographic and clinical characteristics

There were no differences in age, $t(49)=0.18, p>.05$, gender, $\chi^{2}(1)=0.16, p>.05$, or handedness, $\chi^{2}(1)=3.59, p>.05$, between the PO-MDD and healthy groups (see Table 1). As was expected, the PO-MDD group scored higher on average core MDD severity than the healthy group, adjusted $t(44)=$ $10.10, p<.01$ (see Table 1 ). In addition, 18 subjects in the POMDD group ( $=67 \%$ ) showed a history of non-MDD internalizing (i.e., PTSD, GAD, and SAD) and/or externalizing psychiatric symptoms (i.e., ODD, ADHD, and CD).

Group differences in hippocampal volume

Figure 1 illustrates that the PO-MDD group showed significantly smaller left hippocampal volume than did the healthy group, $t(41)=2.17, p<.05$. There was also a trend for the PO-MDD group to show smaller right hippocampal volume than did the healthy group, $t(47)=1.88, p=.07$.
Correlations in the total sample

\section{$50 \%$ and $100 \%$ sad faces}

Left hippocampal volume was negatively correlated with the magnitude of functional activation to sad faces in the left amygdala, right hippocampus, left lateral globus pallidus, right anterior nucleus of the thalamus, right caudate body, and right dorsolateral PFC (DLPFC) (see Table 2 and Fig. 2). These correlations were significant even after controlling for a history of PO-MDD, non-MDD internalizing, and externalizing disorders $(p<.05)$. When the correlations within the ROIs identified in the whole sample were computed within the PO-MDD and healthy control groups separately, the correlations between left hippocampal volume and functional activation in the left amygdala and the right caudate body were significant in both groups (see Table 2). The correlation with functional activation in the left lateral globus pallidus was significant only in the PO-MDD group, whereas the correlations with functional activation in the right hippocampus, right anterior thalamus, and right DLPFC were significant only in healthy controls (see Table 2). However, hierarchical regression analyses indicated that none of correlation magnitudes differed between groups. There were no significant correlations between right hippocampal volume and functional brain responses to sad faces.

\section{$50 \%$ and $100 \%$ all negative faces}

Both left hippocampal (see Fig. 2) and right hippocampal (see Fig. 3) volumes were negatively correlated with left amygdala activation to all negative faces, and these correlations remained significant even after a history of PO-MDD, nonMDD internalizing, and externalizing disorders was covaried $(p<.05)$ (see Table 2). Figure 4 shows scatterplots of correlations between hippocampal volume on each side and left amygdala functional activation. When the correlations within the ROIs detected in the total sample were examined within

Table 1 Demographic and clinical characteristics of the sample

\begin{tabular}{|c|c|c|c|c|}
\hline \multicolumn{2}{|l|}{ Variables } & Total $(N=64)$ & PO-MDD $(n=27)$ & Healthy $(n=24)$ \\
\hline \multicolumn{2}{|l|}{ Age (years)* } & $9.42(1.18)$ & $9.48(1.12)$ & $9.54(1.25)$ \\
\hline \multicolumn{2}{|c|}{ Sex (female/male) } & $36 / 28$ & $15 / 12$ & $12 / 12$ \\
\hline \multicolumn{2}{|c|}{ Handedness (left/right) } & $5 / 59$ & $0 / 27$ & $3 / 21$ \\
\hline \multicolumn{2}{|c|}{ MDD } & 27 & 27 & 0 \\
\hline \multicolumn{2}{|c|}{ Average core MDD score across three annual assessments* } & $2.38(1.64)$ & $3.75(1.27)$ & $0.86(0.65)$ \\
\hline \multirow[t]{2}{*}{ Comorbidity } & Externalizing & $20(9)^{\S}$ & $15(8)^{\S}$ & 0 \\
\hline & Non-MDD Internalizing & $13(9)^{\S}$ & $11(8)^{\S}$ & 0 \\
\hline
\end{tabular}

PO-MDD $=$ history of preschool onset major depressive disorder

*Data presented as mean (standard deviation)

${ }^{\S}$ A value within a parenthesis indicates the number of subjects who showed both externalizing and non-MDD internalizing comorbidities 


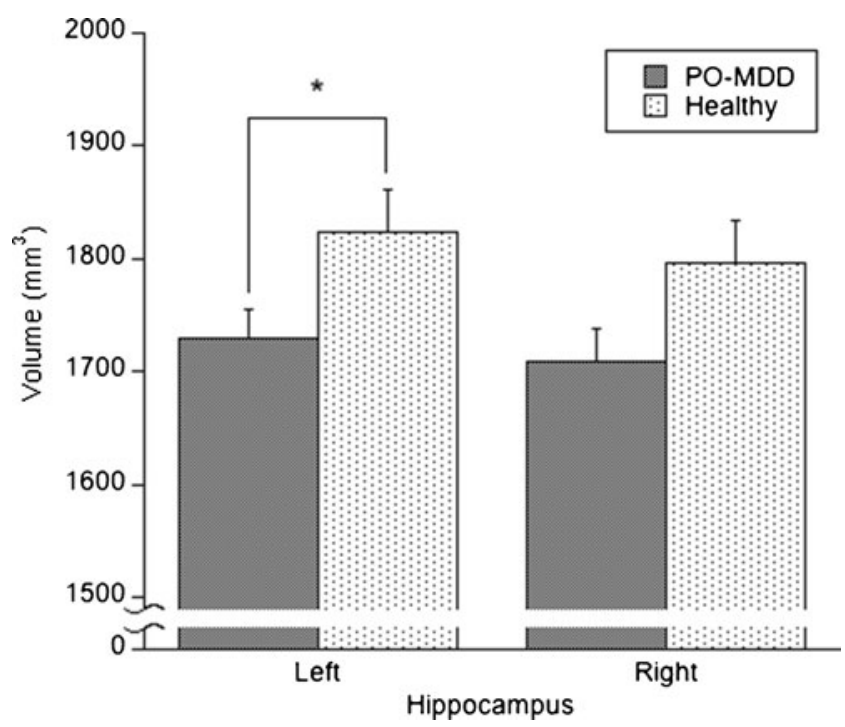

Fig. 1 Comparisons of left and right hippocampal volume between the PO-MDD group and the healthy group. Asterisk indicates a significant difference at $p<.05$

the PO-MDD group and the healthy control group separately, the correlation with left hippocampal volume was significant in both groups (see Table 2). In contrast, the correlation with right hippocampal volume was significant only in the POMDD group, although hierarchical regression analyses indicated that the magnitude of this correlation did not differ between the groups (see Table 2).

\section{Correlations in the PO-MDD group}

Among children with a history of PO-MDD, only right hippocampal volume was negatively correlated with right putamen activation to sad faces and left amygdala activation to all negative faces (see Table 3 and Fig. 5). These correlations remained significant with partialling out a history of non-MDD internalizing and externalizing disorders $(p<.05)$. The healthy controls did not show significant correlations with hippocampal volumes in these same regions (see Table 3). A hierarchical regression analysis revealed that the interaction between group and hippocampal volume was significant in predicting left amygdala activation, indicating that the PO-MDD group showed significantly stronger correlation between right hippocampal volume and left amygdala activation than did the healthy controls (see Table 3). However, the hierarchical regression analysis indicated that there was not a significant group difference in the magnitude of the relationship between right-hippocampal volume and right putamen activity (see Table 3).

Table 2 Functional activity correlations with hippocampal volumes identified in total sample $(N=64)$

\begin{tabular}{|c|c|c|c|c|c|c|c|}
\hline \multirow[t]{2}{*}{ Region of activation ${ }^{\S}$} & \multicolumn{3}{|c|}{ Centroid location $^{\S}$} & \multirow[t]{2}{*}{ \# of voxels ${ }^{\S}$} & \multicolumn{3}{|c|}{ Correlation coefficient } \\
\hline & $X$ & $Y$ & $Z$ & & Total $^{\S}$ & PO-MDD & Healthy \\
\hline \multicolumn{8}{|l|}{ Left hippocampal volume } \\
\hline \multicolumn{8}{|l|}{ Viewing sad faces vs. neutral faces } \\
\hline L Amygdala & -27 & -5 & -13 & 23 & $-.53^{* *}$ & $-.53 *$ & $-.56^{*}$ \\
\hline R Hippocampus & 24 & -40 & 3 & 17 & $-.44 * *$ & -.34 & $-.50 *$ \\
\hline L Lateral Globus Pallidus & -13 & 7 & 0 & 16 & $-.42 * *$ & $-.52 *$ & -.45 \\
\hline R Anterior Nucleus of Thalamus & 8 & -3 & 15 & 17 & $-.46^{* *}$ & -.34 & $-.64 * *$ \\
\hline R Caudate Body & 13 & 9 & 7 & 59 & $-.44^{* *}$ & $-.53^{*}$ & $-.55^{*}$ \\
\hline R Dorsolateral PFC (BA 9) & 36 & 26 & 25 & 35 & $-.43^{* *}$ & -.21 & $-.55^{*}$ \\
\hline \multicolumn{8}{|c|}{ Viewing negative emotional faces vs. neutral faces } \\
\hline L Amygdala & -27 & -2 & -13 & 48 & $-.59 * *$ & $-.64 * *$ & $-.46^{*}$ \\
\hline \multicolumn{8}{|l|}{ Right hippocampal volume } \\
\hline \multicolumn{8}{|l|}{ Viewing sad faces vs. neutral faces } \\
\hline NONE & - & - & - & - & - & - & - \\
\hline \multicolumn{8}{|c|}{ Viewing negative emotional faces vs. neutral faces } \\
\hline L Amygdala & -24 & -10 & -10 & 29 & $-.47 * *$ & $-.50^{*}$ & -.25 \\
\hline
\end{tabular}

According to hierarchical regression analyses, there were no differences in correlation coefficients shown in the table between the PO-MDD and the healthy groups. $\mathrm{R}=$ right hemisphere; $\mathrm{L}=$ left hemisphere. $\mathrm{BA}=$ Brodmann area, $\mathrm{PFC}=$ prefrontal cortex

$* p<.05$

$* * p<.01$

${ }^{\S}$ Data based on analysis of total sample with age and sex as covariates 


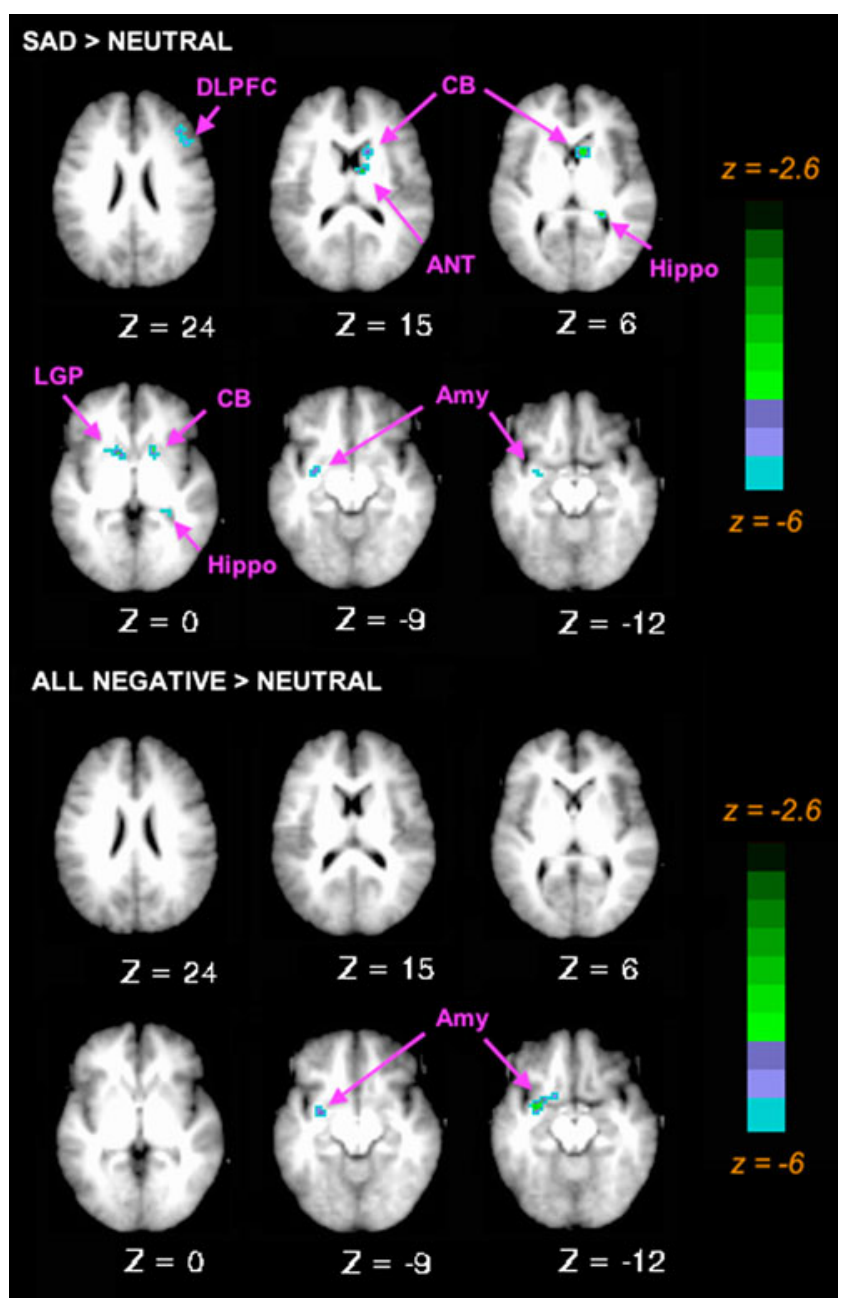

Fig. 2 Brain regions demonstrating correlations between left hippocampal volume and functional brain response to sad faces (upper) or all negative faces (lower) in the total sample. Cool colors represent negative correlations. DLPFC $=$ dorsolateral prefrontal cortex; $\mathrm{ANT}=$ anterior nucleus of thalamus; $\mathrm{CB}=$ caudate body; Hippo = hippocampus; $\mathrm{LGP}=$ lateral globus pallidus; Amy = amygdala

Correlations in healthy controls

In contrast to the PO-MDD group, healthy controls alone demonstrated only one significant correlation, which was between left hippocampal volume and functional brain activity to sad faces in the right anterior nucleus of the thalamus (see Table 4). This correlation was not significant in the PO-MDD group, but a hierarchical regression indicated that the magnitude of this correlation did not differ significantly between the groups (see Table 4).

Group differences in functional activation

As was presented above, the PO-MDD group showed smaller left hippocampal volume (with a trend for reduced right hippocampal volume as well), and we found negative

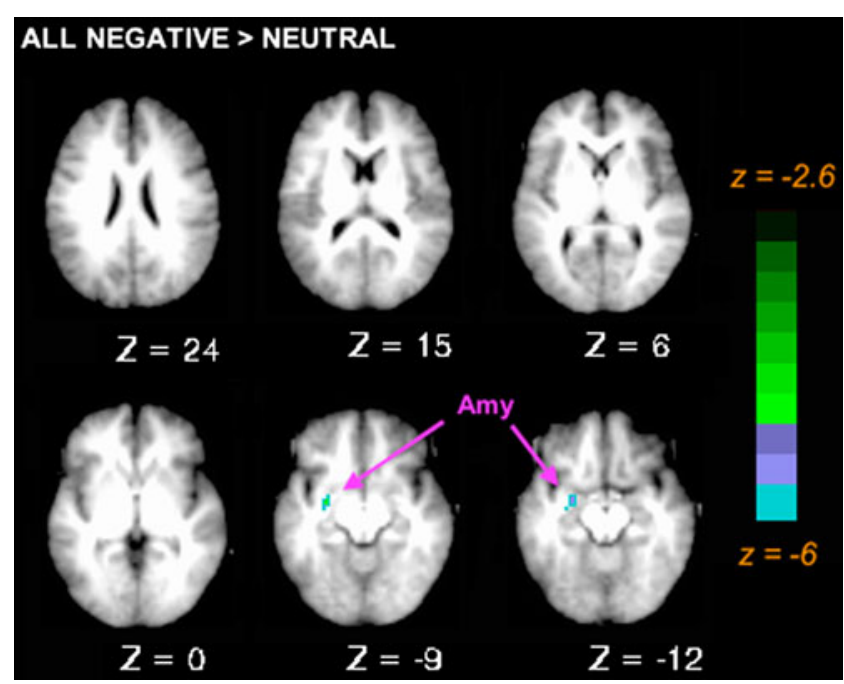

Fig. 3 Brain regions demonstrating correlations between right hippocampal volume and functional brain response to all negative faces in the total sample. Cool colors represent negative correlations. Amy $=$ amygdala

correlations between hippocampal volumes and functional brain activation to negative faces in both the total sample and the PO-MDD group. Thus, we also investigated whether the PO-MDD group showed significantly different activation than did controls in the regions showing correlations with hippocampal volume in the total sample and/or the POMDD analysis only. We computed an ANOVA comparing activation to sad faces versus neutral faces, with group as a between-subjects factor (PO-MDD and healthy) and region (the seven regions from Tables 2 and 3 ) as a within-subjects factor. This ANOVA indicated a trend level main effect of group, $F(1,49)=3.48, p=.07$, but no significant group $\times$ region interaction, $F(6,294)=1.02, p=.80$. The PO-MDD children showed a trend for greater activation than did the healthy children across all regions. We computed a similar ANOVA for all negative faces, as compared with neutral, with group as a between-subjects factor and region as a within-subjects factor. In this case, region had three levels, as shown in Tables 2 and 3. This ANOVA did not reveal any significant main effects or interactions.

\section{Discussion}

The primary goal of the present study was to examine the relationships between hippocampal volume and functional brain activation to sad or negative facial expressions, as compared with neutral expressions, in a sample of children with and without a history of PO-MDD. The present study demonstrated that children with a history of PO-MDD exhibited significantly smaller left hippocampal volume than did healthy controls, with a trend for a similar finding 


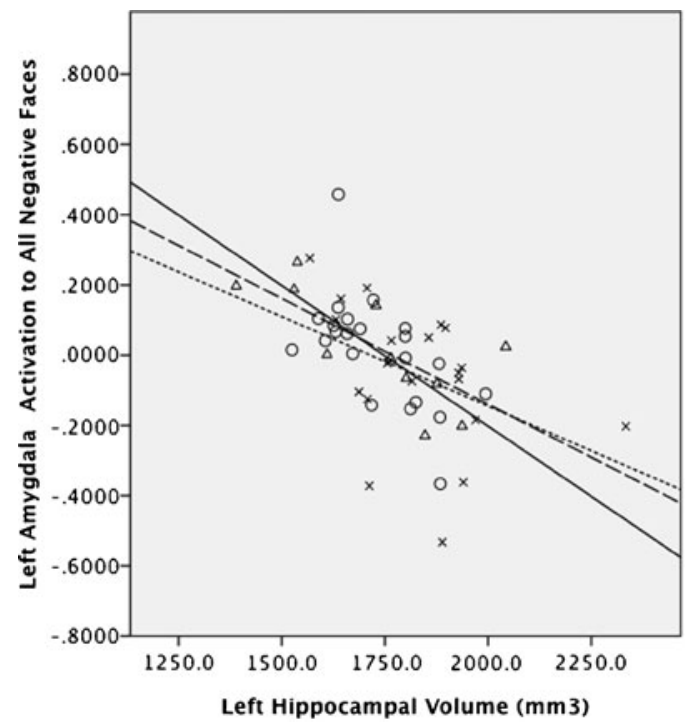

Fig. 4 Scatterplots of correlations between amygdala activation and hippocampal volume. Left scatterplot illustrating the correlation between left hippocampal volume and amygdala responses to all negative faces

in the right hippocampus. These results are consistent with findings in depressed individuals of older ages (BellMcGinty et al., 2002; Bremner et al., 2000; Campbell, Marriott, Nahmias, \& MacQueen, 2004; Frodl et al., 2002; Lorenzetti et al., 2009; MacMaster \& Kusumakar, 2004; MacMaster et al., 2008; Malykhin et al., 2010; McKinnon et al., 2009; O'Brien et al., 2004; Rao et al., 2010; Savitz \& Drevets, 2009; Steffens et al., 2000, 2011; Videbech \& Ravnkilde, 2004). Furthermore, we found that individual differences in hippocampal volume were associated with

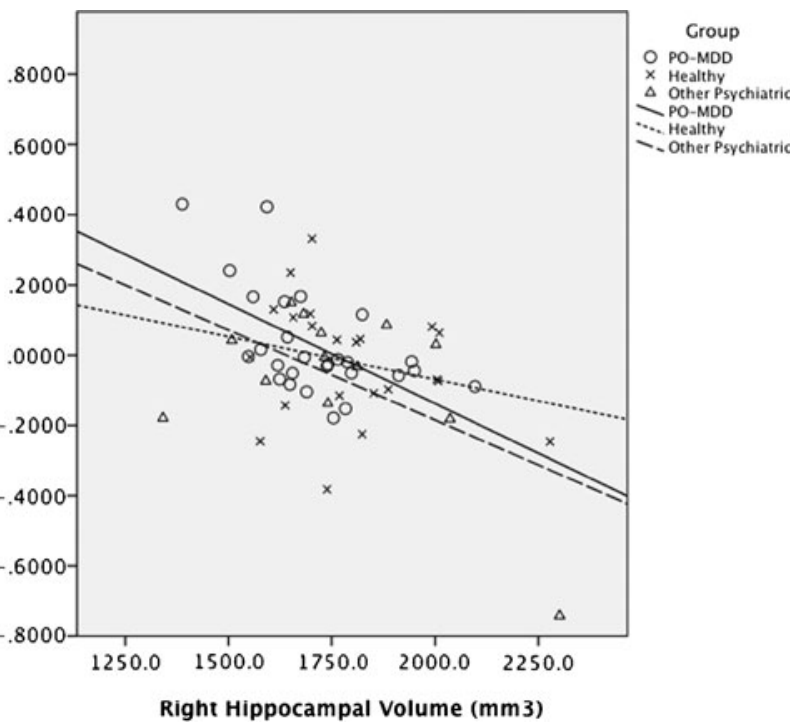

versus neutral faces in the total sample. Right scatterplot illustrating the correlation between right hippocampal volume and amygdala responses to all negative faces versus neutral faces in the total sample

individual differences in functional brain responses to sad or negative faces. Lastly, we found a trend for some of the regions showing correlations between hippocampal volume and functional activation to show enhanced activation in the PO-MDD children, as compared with controls. Taken together, these results provide support for the hypothesis that there is a meaningful relationship between altered hippocampal volume and functional brain activation to affectively valenced stimuli, both in the general population and among children with PO-MDD.

Table 3 Functional activity correlations with hippocampal volumes identified in only PO-MDD $(n=27)$

\begin{tabular}{|c|c|c|c|c|c|c|c|}
\hline \multirow[t]{2}{*}{ Region of activation ${ }^{\S}$} & \multicolumn{3}{|c|}{ Centroid location ${ }^{\S}$} & \multirow[t]{2}{*}{$\#$ of voxels ${ }^{\S}$} & \multicolumn{2}{|c|}{ Correlation coefficient } & \multirow{2}{*}{$\begin{array}{l}\text { Hierarchical regression } \\
\beta\end{array}$} \\
\hline & $X$ & $Y$ & $Z$ & & PO-MDD & Healthy & \\
\hline \multicolumn{8}{|c|}{ Left hippocampal volume } \\
\hline \multicolumn{8}{|c|}{ Viewing sad faces vs. neutral faces } \\
\hline NONE & - & - & - & - & - & - & - \\
\hline \multicolumn{8}{|c|}{ Viewing negative emotional faces vs. neutral faces } \\
\hline NONE & - & - & - & - & - & - & - \\
\hline \multicolumn{8}{|c|}{ Right hippocampal volume } \\
\hline \multicolumn{8}{|c|}{ Viewing sad faces vs. neutral faces } \\
\hline R Putamen & 26 & 2 & 10 & 31 & $-.61 * *$ & -.25 & -1.09 \\
\hline \multicolumn{8}{|c|}{ Viewing negative emotional faces vs. neutral faces } \\
\hline L Amygdala & -16 & -7 & -10 & 30 & $-.70 * *$ & .06 & $-4.77 * *$ \\
\hline
\end{tabular}

The healthy group had $n=22$. The $\beta$ under hierarchical regression is the standardized coefficient for the interaction term of PO-MDD status and hippocampal volume, holding age and sex constant. A significant value indicates group differences in the magnitude of the relationship between hippocampal volume and functional brain response in that region. $\mathrm{R}=$ right hemisphere; $\mathrm{L}=$ left hemisphere

$* * p<.01$

${ }^{\S}$ Data based on analysis of the never-medicated MDD group 


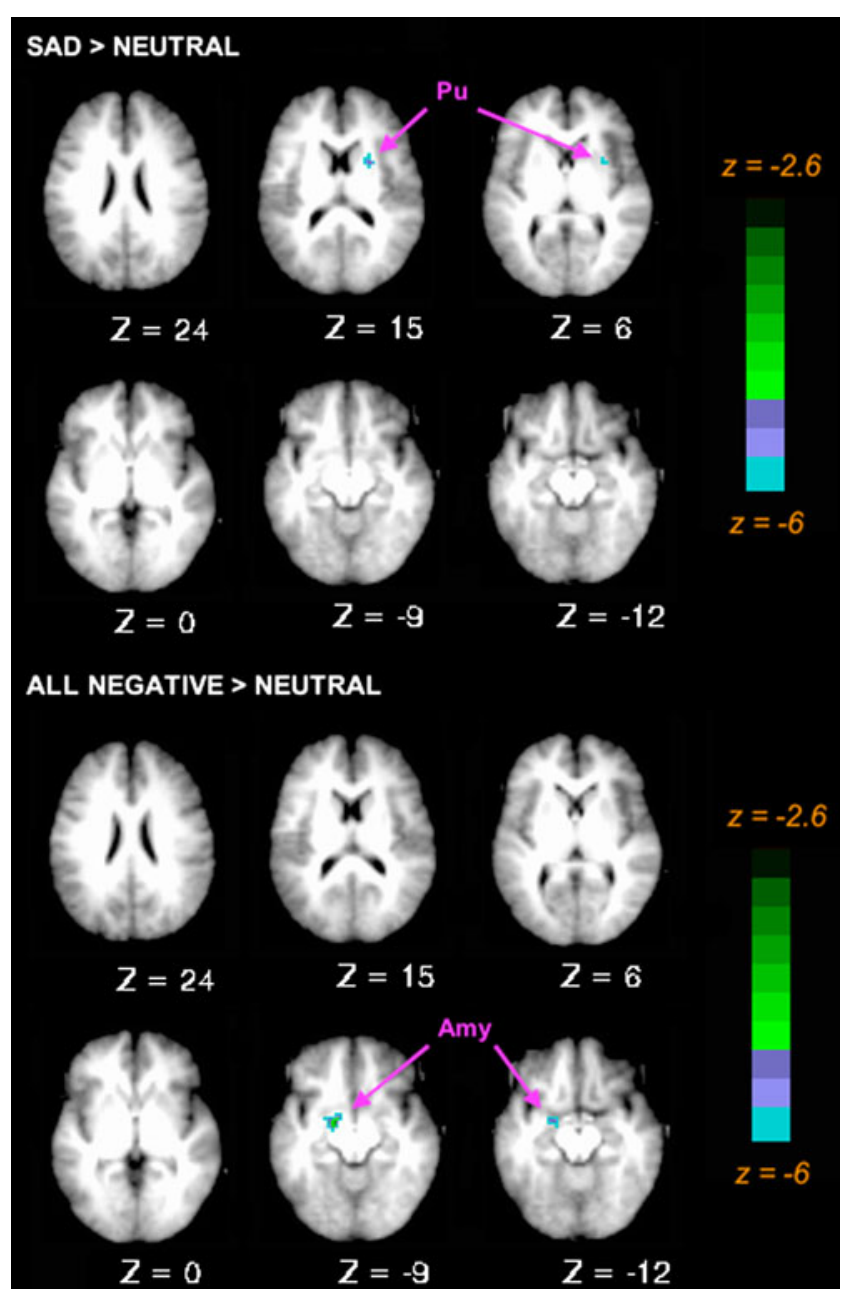

Fig. 5 Brain regions demonstrating correlations between right hippocampal volume and functional brain response to sad faces (upper) or all negative faces (lower) in the PO-MDD sample. Cool colors represent negative correlations. $\mathrm{Pu}=$ putamen; $\mathrm{Amy}=$ amygdala

Hippocampal volume and functional brain responses to negative faces in the whole sample

The results of analyses in the total sample indicated that greater left hippocampal volume was associated with less activation to sad faces (as compared with neutral) in the left amygdala, right (posterior) hippocampus, left globus pallidus, right anterior nucleus of the thalamus, right caudate, and right DLPFC. In contrast, there were no such relationships with right hippocampal volume in the total sample. However, greater right and left hippocampal volumes were both associated with less left amygdala activation to all negative faces versus neutral faces. Furthermore, almost all of the correlations with the left hippocampus remained significant when examined separately in the PO-MDD and healthy groups. The fact that these relationships were similar in both the PO-MDD and healthy groups suggests that, at least, some of the mechanisms driving the relationships between hippocampal volume and functional brain activation to emotional valenced faces are not specific or unique to MDD.

It is particularly interesting that left hippocampal volume was associated with functional reactivity in the left amygdala, right posterior hippocampus, and right DLPFC in response to sad facial expressions. These regions have been consistently implicated as important for emotional reactivity and regulation (Mayberg, 2003). Specifically, the amygdala responds to a wide variety of negative stimuli (Fusar-Poli et al., 2009; Whalen et al., 2001), consistent with the hypothesis that the amygdala functions to help perceive affective salience or signals in the environment (Tottenham \& Sheridan, 2009). Episodic encoding of emotional stimuli may be performed by the hippocampus, as evidenced by studies showing increased activation in the hippocampus during emotional/facial encoding (Chua, Schacter, RandGiovannetti, \& Sperling, 2007; Fischer, Nyberg, \& Backman, 2010; Ku, Tolias, Logothetis, \& Goense, 2011). However, encoding of emotional stimuli increases functional activation in the anterior, rather than posterior, subregion of the hippocampus (Dolcos et al., 2004; Small et al., 2011), whereas the present study found that altered hippocampal volume was associated with functional activity in a more posterior aspect of the hippocampus in response to sad faces. Prior data suggest that the posterior hippocampus serves to help support processing of and memory for emotionally neutral stimuli (Dolcos et al., 2004; Small et al., 2011). Additional research is needed to confirm these distinctions in the processing functions of anterior versus posterior hippocampal regions and to replicate our findings of correlations between hippocampal volume and activation to emotional faces in posterior, rather than anterior, region.

Some aspects of emotional processing in the amygdala and hippocampus are regulated by the DLPFC (Maletic et al., 2007; Phillips et al., 2003). For example, healthy individuals showed increased activation in the right DLPFC when they were instructed to ignore fearful stimuli (Fales et al., 2008). Nevertheless, given that depression is thought to be associated with reduced hippocampal volume (Caetano et al., 2007; MacMaster \& Kusumakar, 2004; MacMaster et al., 2008; Rao et al., 2010) and less activation to negative stimuli in the DLPFC (Hamilton et al., 2012), it was unexpected that the present study found the negative relationship between hippocampal volume and DLPFC activation. As such, this finding needs to be replicated and expanded in future work.

Taken together, the results of the present study are consistent with the hypothesis that left hippocampal structural integrity (as indexed by volume) may modulate emotional responsivity in regions such as the left amygdala and the right hippocampus, as well as potentially influence emotional regulation functions subserved by the DLPFC. An alternative mechanism could be that hypersensitivity to negative 
Table 4 Functional activity correlations with hippocampal volume identified in only healthy controls $(n=24)$

\begin{tabular}{|c|c|c|c|c|c|c|c|}
\hline \multirow[t]{2}{*}{ Region of activation ${ }^{\S}$} & \multicolumn{3}{|c|}{ Centroid location ${ }^{\S}$} & \multirow[t]{2}{*}{$\#$ of voxels ${ }^{\S}$} & \multicolumn{2}{|c|}{ Correlation coefficient } & \multirow{2}{*}{$\begin{array}{l}\text { Hierarchical regression } \\
\beta\end{array}$} \\
\hline & $X$ & $Y$ & $Z$ & & PO-MDD & Healthy & \\
\hline \multicolumn{8}{|l|}{ Left hippocampal volume } \\
\hline \multicolumn{8}{|l|}{ Viewing sad faces vs. neutral faces } \\
\hline R Anterior Nucleus of Thalamus & 7 & -3 & 15 & 16 & -.23 & $-.66^{* *}$ & 1.70 \\
\hline \multicolumn{8}{|c|}{ Viewing negative emotional faces vs. neutral faces } \\
\hline NONE & - & - & - & - & - & - & - \\
\hline \multicolumn{8}{|l|}{ Right hippocampal volume } \\
\hline \multicolumn{8}{|l|}{ Viewing sad faces vs. neutral faces } \\
\hline NONE & - & - & - & - & - & - & - \\
\hline \multicolumn{8}{|c|}{ Viewing negative emotional faces vs. neutral faces } \\
\hline NONE & - & - & - & - & - & - & - \\
\hline
\end{tabular}

The MDD group had $n=27$. The $\beta$ under hierarchical regression is the standardized coefficient for the interaction term of PO-MDD status and hippocampal volume, holding age and sex constant. A significant value indicates group differences in the magnitude of the relationship between hippocampal volume and functional brain response in that region. $\mathrm{R}=$ right hemisphere

$* * p<.01$

$\S^{\S}$ Data based on analysis of the never-medicated MDD group

stimuli in regions such as the amygdala or the DLPFC might influence the structure of the hippocampus, through excitotoxic effects of glutamate accumulation (Sapolsky, 2000b) or repeated activation of the HPA axis (Juster, McEwen, \& Lupien, 2010; McEwen, Eiland, Hunter, \& Miller, 2012) that might contribute to loss of or inhibited neurogenesis or synaptogenesis of neurons, leading to hippocampal atrophy.

PO-MDD-specific relationships between hippocampal volume and functional brain responses to negative faces

The results of analyses in the PO-MDD group alone yielded significant correlations between right hippocampal volume (but not left) and functional activation to sad faces in the right putamen and to all negative faces in the left amygdala. In particular, the correlation between right hippocampal volume and left amygdala activation was (1) not identified in the healthy controls, (2) remained significant even when controlling for a history of non-MDD psychiatric disorders, and (3) was significantly stronger in the PO-MDD than in the healthy group. Such results suggest that the negative correlation between right hippocampal volume and left amygdala activation may be specific to PO-MDD, rather than generalized to internalizing or externalizing psychopathology.

Reduced hippocampal volume (Caetano et al., 2007; MacMaster \& Kusumakar, 2004; MacMaster et al., 2008; Rao et al., 2010), as well as increased amygdala responses to negative stimuli (Barch et al., in press; Gaffrey et al. 2011b; Roberson-Nay et al., 2006), has been found in childhood and/ or adolescent MDD. Our findings are consistent with and help elucidate the relationship between these two biomarkers for
MDD. There is a discrepancy across fMRI findings such that depressed adolescents have shown increased functional activation to emotionally evocative stimuli (Roberson-Nay et al., 2006) but decreased activation to fearful faces (Thomas et al., 2001) in the left amygdala. Although our findings do not clearly help us to understand such discrepancies in the literature, it would be interesting to determine whether variability in amygdala responsivity across prior studies was related to hippocampal volume differences across studies.

The correlation between right hippocampal volume and functional activation to sad faces in the right putamen was also found in the PO-MDD group, but not the whole sample or the healthy controls, even when controlling for a history of non-MDD psychiatric disorders. However, these findings provided only weak evidence for a specific PO-MDD finding because the strength of this correlation in the PO-MDD group did not significantly differ from that in the controls. Nevertheless, one interesting study showed that 8-year-old children with potential high risk for MDD (i.e., T allele carriers of CERB1 gene) showed increased activation in the right putamen, as compared with controls (i.e., $\mathrm{C}$ allele carriers), when they viewed sad-film clips (Fortier et al., 2011). Hence, hyperactivation to sad stimuli in the right putamen may be associated with risk for MDD, and our study additionally suggests that this activation in the right putamen may be related to right hippocampal volume.

Limitations

The analyses in the total sample used a modest sample size $(N=64)$ that is comparable to or larger than sample sizes 
used in many other child-focused studies. However, the sample sizes for the PO-MDD and control groups alone were smaller, reducing the power for analyses in these subgroups. Related to this point, the significant correlations with left hippocampal volume found in the whole sample (i.e., correlations between left hippocampal volume and functional activation to sad/all-negative faces in the left amygdala, left lateral globus pallidus, and right caudate body) also remained significant when tested within the PO-MDD group at the ROI level. However, these correlations were not identified in the voxel-wise analyses with the PO-MDD group alone. This discrepancy likely reflects the smaller sample size for the PO-MDD group $(n=27)$, as compared with the whole sample, which reduced statistical power to detect the correlations in the voxel-by-voxel analysis in the PO-MDD group alone.

A second limitation is that our results cannot inform the direction of effects (e.g., determine whether reductions in hippocampal volume contribute to increased functional brain activation or vice versa). As was discussed above, some studies in adults have indicated that reduced hippocampal volume was correlated with longer MDD episode durations (Bell-McGinty et al., 2002; Colla et al., 2007; MacQueen et al., 2003; McKinnon et al., 2009; Sheline et al., 1996, 1999). These findings contribute to the argument that hippocampal volume reduction may result from the experience of MDD, including potentially hyperactive emotional responses. It will be necessary to conduct longitudinal investigations to clarify the nature of the causal relationships between hippocampal atrophy and altered functional activation in the brain.

\section{Conclusions}

In summary, the present findings demonstrated that children with PO-MDD showed smaller left hippocampal volume than did healthy children, that left hippocampal volume was associated with functional brain activation to negative faces in both PO-MDD and healthy children, that right hippocampal volume was associated with functional brain activation to negative faces in PO-MDD children alone, and that the regions showing such correlations with hippocampal volume tended to show enhanced activation in the POMDD children, as compared with the healthy children. Taken together, these findings provide direct evidence that there may be common mechanisms contributing to hippocampal volume reductions and hyperactivity of corticolimbic regions in response to negative stimuli among children with PO-MDD. These data cannot inform us as to the direction of these relationships, and further work will be needed to determine whether reduced hippocampal volume contributes to hyperresponsivity of cortico-limbic regions to emotional stimuli, whether hyperresponsivity to emotional stimuli contributes to hippocampal volume reduction, or whether another variable critical to the pathophysiology of MDD contributes to both reduced hippocampal volume and increased cortico-limbic activity to negative stimuli.

Author note This study was supported by NIH Grants R01MH64769 (J.L.L.), R01-MH090786 (J.L.L., D.M.B., K.N.B.), R01EB008171 (M.I.M., J.T.R., K.N.B.), and P41-EB015909 (M.I.M., J.T.R.). The authors would like thank the families and children who participated in these studies, and the authors would like to give special thanks to Rebecca Tillman, Laura Rayhawk, Ted Handler, Jessica Stachecki, and other EEDP staffs because of their immense assistance for our research project.

\section{References}

Anagnostaras, S. G., Maren, S., \& Fanselow, M. S. (1999). Temporally graded retrograde amnesia of contextual fear after hippocampal damage in rats: Within-subjects examination. Journal of Neuroscience, 19(3), 1106-1114.

Barch, D. M., Gaffrey, M. S., Botteron, K. N., Belden, A. C., \& Luby, J. L. (in press). Functional brain activation to emotionally valenced faces in school aged children with a history of preschool onset major depression. Biology Psychiatry.

Becerril, K. E., Repovs, G., \& Barch, D. M. (2011). Error processing network dynamics in schizophrenia. NeuroImage, 54(2), 14951505. doi:10.1016/J.Neuroimage.2010.09.046

Beg, M. F., Miller, M. I., Trouve, A., \& Younes, L. (2005). Computing large deformation metric mappings via geodesic flows of diffeomorphisms. International Journal of Computer Vision, 61(2), $139-157$.

Bell-McGinty, S., Butters, M. A., Meltzer, C. C., Greer, P. J., Reynolds, C. F., 3rd, \& Becker, J. T. (2002). Brain morphometric abnormalities in geriatric depression: Long-term neurobiological effects of illness duration. The American Journal of Psychiatry, 159(8), $1424-1427$.

Blugeot, A., Rivat, C., Bouvier, E., Molet, J., Mouchard, A., Zeau, B., \& Becker, C. (2011). Vulnerability to depression: From brain neuroplasticity to identification of biomarkers. Journal of Neuroscience, 31(36), 12889-12899. doi:10.1523/JNEUROSCI.130911.2011

Botteron, K. N. (2001). Genetic analysis of brain imaging abnormalities. Child and Adolescent Psychiatric Clinics of North America, 10(2), 241-258. viii.

Braver, T. S., Paxton, J. L., Locke, H. S., \& Barch, D. M. (2009). Flexible neural mechanisms of cognitive control within human prefrontal cortex. Proceedings of the National Academy of Sciences of the United States of America, 106(18), 7351-7356. doi:10.1073/Pnas.0808187106

Bremner, J. D., Narayan, M., Anderson, E. R., Staib, L. H., Miller, H. L., \& Charney, D. S. (2000). Hippocampal volume reduction in major depression. The American Journal of Psychiatry, 157(1), $115-118$

Buckner, R. L., Head, D., Parker, J., Fotenos, A. F., Marcus, D., Morris, J. C., \& Snyder, A. Z. (2004). A unified approach for morphometric and functional data analysis in young, old, and demented adults using automated atlas-based head size normalization: Reliability and validation against manual measurement of total intracranial volume. NeuroImage, 23(2), 724-738. doi:10.1016/j.neuroimage.2004.06.018 
Burgund, E. D., Kang, H. C., Kelly, J. E., Buckner, R. L., Snyder, A. Z., Petersen, S. E., \& Schlaggar, B. L. (2002). The feasibility of a common stereotactic space for children and adults in fMRI studies of development. NeuroImage, 17(1), 184-200.

Burke, H. M., Davis, M. C., Otte, C., \& Mohr, D. C. (2005). Depression and cortisol responses to psychological stress: A metaanalysis. Psychoneuroendocrinology, 30(9), 846-856. doi:10.1016/j.psyneuen.2005.02.010

Caetano, S. C., Fonseca, M., Hatch, J. P., Olvera, R. L., Nicoletti, M., Hunter, K., \& Soares, J. C. (2007). Medial temporal lobe abnormalities in pediatric unipolar depression. Neuroscience Letters, 427(3), 142-147. doi:10.1016/j.neulet.2007.06.014

Campbell, S., Marriott, M., Nahmias, C., \& MacQueen, G. M. (2004). Lower hippocampal volume in patients suffering from depression: A meta-analysis. The American Journal of Psychiatry, 161(4), 598-607.

Chua, E. F., Schacter, D. L., Rand-Giovannetti, E., \& Sperling, R. A. (2007). Evidence for a specific role of the anterior hippocampal region in successful associative encoding. Hippocampus, 17(11), 1071-1080. doi:10.1002/hipo.20340

Colla, M., Kronenberg, G., Deuschle, M., Meichel, K., Hagen, T., Bohrer, M., \& Heuser, I. (2007). Hippocampal volume reduction and HPA-system activity in major depression. Journal of Psychiatric Research, 41(7), 553-560. doi:10.1016/ j.jpsychires.2006.06.011

Csernansky, J. G., Joshi, S., Wang, L., Haller, J. W., Gado, M., Miller, J. P., \& Miller, M. I. (1998). Hippocampal morphometry in schizophrenia by high dimensional brain mapping. Proceedings of the National Academy of Sciences of the United States of America, 95(19), 11406-11411.

Dolcos, F., LaBar, K. S., \& Cabeza, R. (2004). Interaction between the amygdala and the medial temporal lobe memory system predicts better memory for emotional events. Neuron, 42(5), 855-863.

Drevets, W. C., Price, J. L., \& Furey, M. L. (2008). Brain structural and functional abnormalities in mood disorders: Implications for neurocircuitry models of depression. Brain Structure \& Function, 213(1-2), 93-118. doi:10.1007/s00429-008-0189-x

Egger, H. L. (2009). Psychiatric assessment of young children. Child and Adolescent Psychiatric Clinics of North America, 18(3), 559580. doi:10.1016/j.chc.2009.02.004

Egger, H., Ascher, B., \& Angold, A. (1999, 2003). The Preschool Age Psychiatric Assessment: Version 1.4. Duke University Medical Center, Durham, NC.

Egger, H. L., Erkanli, A., Keeler, G., Potts, E., Walter, B. K., \& Angold, A. (2006). Test-Retest Reliability of the Preschool Age Psychiatric Assessment (PAPA). Journal of the American Academy of Child and Adolescent Psychiatry, 45(5), 538-549. doi:10.1097/01.chi.0000205705.71194.b8

Fales, C. L., Barch, D. M., Rundle, M. M., Mintun, M. A., Mathews, J., Snyder, A. Z., \& Sheline, Y. I. (2009). Antidepressant treatment normalizes hypoactivity in dorsolateral prefrontal cortex during emotional interference processing in major depression. Journal of Affective Disorders, 112(1-3), 206-211. doi:10.1016/ j.jad.2008.04.027

Fales, C. L., Barch, D. M., Rundle, M. M., Mintun, M. A., Snyder, A. Z., Cohen, J. D., \& Sheline, Y. I. (2008). Altered emotional interference processing in affective and cognitive-control brain circuitry in major depression. Biological Psychiatry, 63(4), 377384. doi:10.1016/j.biopsych.2007.06.012

Fischer, H., Nyberg, L., \& Backman, L. (2010). Age-related differences in brain regions supporting successful encoding of emotional faces. Cortex, 46(4), 490-497. doi:10.1016/ j.cortex.2009.05.011

Forman, S. D., Cohen, J. D., Fitzgerald, M., Eddy, W. F., Mintun, M. A., \& Noll, D. C. (1995). Improved assessment of significant activation in functional magnetic-resonance-imaging (Fmri) - use of a cluster-size threshold. Magnetic Resonance in Medicine, 33(5), 636-647.

Fortier, E., Noreau, A., Lepore, F., Boivin, M., Perusse, D., Rouleau, G. A., \& Beauregard, M. (2011). Early influence of the rs 4675690 on the neural substrates of sadness. Journal of Affective Disorders. doi:10.1016/j.jad.2011.06.039

Frodl, T., Meisenzahl, E. M., Zetzsche, T., Born, C., Groll, C., Jager, M., \& Moller, H. J. (2002). Hippocampal changes in patients with a first episode of major depression. The American Journal of Psychiatry, 159(7), 1112-1118.

Frodl, T., Schaub, A., Banac, S., Charypar, M., Jager, M., Kummler, P., \& Meisenzahl, E. M. (2006). Reduced hippocampal volume correlates with executive dysfunctioning in major depression. Journal of Psychiatry \& Neuroscience, 31(5), 316-323.

Fu, C. H., Williams, S. C., Cleare, A. J., Brammer, M. J., Walsh, N. D., Kim, J., \& Bullmore, E. T. (2004). Attenuation of the neural response to sad faces in major depression by antidepressant treatment: A prospective, event-related functional magnetic resonance imaging study. Archives of General Psychiatry, 61(9), 877-889. doi:10.1001/archpsyc.61.9.877

Fusar-Poli, P., Placentino, A., Carletti, F., Landi, P., Allen, P., Surguladze, S., \& Politi, P. (2009). Functional atlas of emotional faces processing: A voxel-based meta-analysis of 105 functional magnetic resonance imaging studies. Journal of Psychiatry \& Neuroscience, 34 (6), 418-432.

Gaffrey, M. S., Belden, A. C., \& Luby, J. L. (2011a). The 2-week duration criterion and severity and course of early childhood depression: Implications for nosology. Journal of Affective Disorders, 133(3), 537-545. doi:10.1016/j.jad.2011.04.056

Gaffrey, M. S., Luby, J. L., Belden, A. C., Hirshberg, J. S., Volsch, J., \& Barch, D. M. (2011b). Association between depression severity and amygdala reactivity during sad face viewing in depressed preschoolers: An fMRI study. Journal of Affective Disorders, 129(1-3), 364-370. doi:10.1016/J.Jad.2010.08.031

Haller, J. W., Banerjee, A., Christensen, G. E., Gado, M., Joshi, S., Miller, M. I., \& Csernansky, J. G. (1997). Threedimensional hippocampal MR morphometry with highdimensional transformation of a neuroanatomic atlas. Radiology, 202(2), 504-510.

Haller, J. W., Christensen, G. E., Joshi, S. C., Newcomer, J. W., Miller, M. I., Csernansky, J. G., \& Vannier, M. W. (1996). Hippocampal MR imaging morphometry by means of general pattern matching. Radiology, 199(3), 787-791.

Hamilton, J. P., Etkin, A., Furman, D. J., Lemus, M. G., Johnson, R. F., \& Gotlib, I. H. (2012). Functional neuroimaging of major depressive disorder: A meta-analysis and new integration of baseline activation and neural response data. The American Journal of Psychiatry. doi:10.1176/appi.ajp.2012.11071105

Holmes, C. J., Hoge, R., Collins, L., Woods, R., Toga, A. W., \& Evans, A. C. (1998). Enhancement of MR images using registration for signal averaging. Journal of Computer Assisted Tomography, 22 (2), 324-333.

Ji, J., \& Maren, S. (2007). Hippocampal involvement in contextual modulation of fear extinction. Hippocampus, 17(9), 749-758. doi:10.1002/hipo.20331

Jimura, K., Locke, H. S., \& Braver, T. S. (2010). Prefrontal cortex mediation of cognitive enhancement in rewarding motivational contexts. Proceedings of the National Academy of Sciences of the United States of America, 107(19), 8871-8876. doi:10.1073/ Pnas. 1002007107

Juster, R. P., McEwen, B. S., \& Lupien, S. J. (2010). Allostatic load biomarkers of chronic stress and impact on health and cognition. Neuroscience and Biobehavioral Reviews, 35(1), 2-16. doi:10.1016/j.neubiorev.2009.10.002

Kang, H. C., Burgund, E. D., Lugar, H. M., Petersen, S. E., \& Schlaggar, B. L. (2003). Comparison of functional activation foci 
in children and adults using a common stereotactic space. NeuroImage, 19(1), 16-28.

Karl, A., Schaefer, M., Malta, L. S., Dorfel, D., Rohleder, N., \& Werner, A. (2006). A meta-analysis of structural brain abnormalities in PTSD. Neuroscience and Biobehavioral Reviews, 30(7), 1004-1031. doi:10.1016/j.neubiorev.2006.03.004

Ku, S. P., Tolias, A. S., Logothetis, N. K., \& Goense, J. (2011). fMRI of the face-processing network in the ventral temporal lobe of awake and anesthetized macaques. Neuron, 70(2), 352-362. doi:10.1016/j.neuron.2011.02.048

Lopez-Duran, N. L., Kovacs, M., \& George, C. J. (2009). Hypothalamic-pituitary-adrenal axis dysregulation in depressed children and adolescents: A meta-analysis. Psychoneuroendocrinology, 34(9), 1272-1283. doi:10.1016/j.psyneuen.2009.03.016

Lorenzetti, V., Allen, N. B., Fornito, A., \& Yucel, M. (2009). Structural brain abnormalities in major depressive disorder: A selective review of recent MRI studies. Journal of Affective Disorders, 117(1-2), 1-17. doi:10.1016/j.jad.2008.11.021

Luby, J. L., Belden, A. C., Pautsch, J., Si, X., \& Spitznagel, E. (2009a). The clinical significance of preschool depression: Impairment in functioning and clinical markers of the disorder. Journal of Affective Disorders, 112(1-3), 111-119. doi:10.1016/j.jad.2008.03.026

Luby, J., Belden, A., Sullivan, J., Hayen, R., McCadney, A., \& Spitznagel, E. (2009b). Shame and guilt in preschool depression: Evidence for elevations in self-conscious emotions in depression as early as age 3 . Journal of Child Psychology and Psychiatry, 50(9), 1156-1166. doi:10.1111/j.1469-7610.2009.02077.x

Luby, J. L., Heffelfinger, A., Mrakotsky, C., Brown, K., Hessler, M., \& Spitznagel, E. (2003a). Alterations in stress cortisol reactivity in depressed preschoolers relative to psychiatric and no-disorder comparison groups. Archives of General Psychiatry, 60(12), 1248-1255. doi:10.1001/archpsyc.60.12.1248

Luby, J. L., Heffelfinger, A. K., Mrakotsky, C., Hessler, M. J., Brown, K. M., \& Hildebrand, T. (2002). Preschool major depressive disorder: Preliminary validation for developmentally modified DSM-IV criteria. Journal of the American Academy of Child and Adolescent Psychiatry, 41(8), 928-937. doi:10.1097/ 00004583-200208000-00011

Luby, J. L., Mrakotsky, C., Heffelfinger, A., Brown, K., Hessler, M., \& Spitznagel, E. (2003b). Modification of DSM-IV criteria for depressed preschool children. The American Journal of Psychiatry, 160(6), 1169-1172.

Luby, J. L., Mrakotsky, C., Heffelfinger, A., Brown, K., \& Spitznagel, E. (2004). Characteristics of depressed preschoolers with and without anhedonia: Evidence for a melancholic depressive subtype in young children. The American Journal of Psychiatry, 161 (11), 1998-2004. doi:10.1176/appi.ajp.161.11.1998

Luby, J. L., Si, X., Belden, A. C., Tandon, M., \& Spitznagel, E. (2009c). Preschool depression: Homotypic continuity and course over 24 months. Archives of General Psychiatry, 66(8), 897-905. doi:10.1001/archgenpsychiatry.2009.97

Luby, J. L., Sullivan, J., Belden, A., Stalets, M., Blankenship, S., \& Spitznagel, E. (2006). An observational analysis of behavior in depressed preschoolers: Further validation of early-onset depression. Journal of the American Academy of Child and Adolescent Psychiatry, 45(2), 203-212. doi:10.1097/01.chi.0000188894.54713.ee

Luking, K. R., Repovs, G., Belden, A. C., Gaffrey, M. S., Botteron, K. N., Luby, J. L., \& Barch, D. M. (2011). Functional connectivity of the amygdala in early-childhood-onset depression. Journal of the American Academy of Child and Adolescent Psychiatry, 50(10), 1027-1041. doi:10.1016/j.jaac.2011.07.019

Machado, C. J., \& Bachevalier, J. (2006). The impact of selective amygdala, orbital frontal cortex, or hippocampal formation lesions on established social relationships in rhesus monkeys (Macaca mulatta). Behavioral Neuroscience, 120(4), 761-786. doi:10.1037/0735-7044.120.4.761
MacMaster, F. P., \& Kusumakar, V. (2004). Hippocampal volume in early onset depression. BMC Medicine, 2, 2. doi:10.1186/17417015-2-2

MacMaster, F. P., Mirza, Y., Szeszko, P. R., Kmiecik, L. E., Easter, P. C., Taormina, S. P., \& Rosenberg, D. R. (2008). Amygdala and hippocampal volumes in familial early onset major depressive disorder. Biological Psychiatry, 63(4), 385-390. doi:10.1016/ j.biopsych.2007.05.005

MacMillan, S., Szeszko, P. R., Moore, G. J., Madden, R., Lorch, E., Ivey, J., \& Rosenberg, D. R. (2003). Increased amygdala: Hippocampal volume ratios associated with severity of anxiety in pediatric major depression. Journal of Child and Adolescent Psychopharmacology, 13(1), 65-73. doi:10.1089/ 104454603321666207

MacQueen, G. M., Campbell, S., McEwen, B. S., Macdonald, K., Amano, S., Joffe, R. T., \& Young, L. T. (2003). Course of illness, hippocampal function, and hippocampal volume in major depression. Proceedings of the National Academy of Sciences of the United States of America, 100(3), 1387-1392. doi:10.1073/ pnas. 0337481100

Maletic, V., Robinson, M., Oakes, T., Iyengar, S., Ball, S. G., \& Russell, J. (2007). Neurobiology of depression: An integrated view of key findings. International Journal of Clinical Practice, 61(12), 2030-2040. doi:10.1111/j.1742-1241.2007.01602.x

Malykhin, N. V., Carter, R., Seres, P., \& Coupland, N. J. (2010). Structural changes in the hippocampus in major depressive disorder: Contributions of disease and treatment. Journal of Psychiatry \& Neuroscience, 35(5), 337-343. doi:10.1503/jpn.100002

Mamah, D., Wang, L., Barch, D., de Erausquin, G. A., Gado, M., \& Csernansky, J. G. (2007). Structural analysis of the basal ganglia in schizophrenia. Schizophrenia Research, 89(1-3), 59-71.

Mamah, D., Wang, L., Csernansky, J. G., Rice, J. P., Smith, M., \& Barch, D. M. (2010). Morphometry of the hippocampus and amygdala in bipolar disorder and schizophrenia. Bipolar Disorders, 12(3), 341-343. doi:10.1111/j.1399-5618.2010.00802.x

Mayberg, H. S. (2003). Modulating dysfunctional limbic-cortical circuits in depression: Towards development of brain-based algorithms for diagnosis and optimised treatment. British Medical Bulletin, 65, 193-207.

McAvoy, M. P., Ollinger, J. M., \& Buckner, R. L. (2001). Cluster size thresholds for assessment of significant activation in fMRI. NeuroImage, 13(6), S198-S198.

McEwen, B. S. (2000). Effects of adverse experiences for brain structure and function. Biological Psychiatry, 48(8), 721-731.

McEwen, B. S., Eiland, L., Hunter, R. G., \& Miller, M. M. (2011). Stress and anxiety: Structural plasticity and epigenetic regulation as a consequence of stress. Neuropharmacology. doi:10.1016/ j.neuropharm.2011.07.014

McEwen, B. S., Eiland, L., Hunter, R. G., \& Miller, M. M. (2012). Stress and anxiety: Structural plasticity and epigenetic regulation as a consequence of stress. Neuropharmacology, 62(1), 3-12. doi:10.1016/j.neuropharm.2011.07.014

McKinnon, M. C., Yucel, K., Nazarov, A., \& MacQueen, G. M. (2009). A meta-analysis examining clinical predictors of hippocampal volume in patients with major depressive disorder. Journal of Psychiatry \& Neuroscience, 34(1), 41-54.

Miller, M. I., Trouve, A., \& Younes, L. (2002). On the metrics and euler-lagrange equations of computational anatomy. Annual Review of Biomedical Engineering, 4, 375-405. doi:10.1146/ annurev.bioeng.4.092101.125733

Monk, C. S., Klein, R. G., Telzer, E. H., Schroth, E. A., Mannuzza, S., Moulton, J. L., 3rd, \& Ernst, M. (2008). Amygdala and nucleus accumbens activation to emotional facial expressions in children and adolescents at risk for major depression. The American Journal of Psychiatry, 165(1), 90-98. doi:10.1176/ appi.ajp.2007.06111917 
O'Brien, J. T., Lloyd, A., McKeith, I., Gholkar, A., \& Ferrier, N. (2004). A longitudinal study of hippocampal volume, cortisol levels, and cognition in older depressed subjects. The American Journal of Psychiatry, 161(11), 2081-2090. doi:10.1176/ appi.ajp.161.11.2081

Ojemann, J., Akbudak, E., Snyder, A., McKinstry, R., Raichle, M., \& Conturo, T. (1997). Anatomic localization and quantitative analysis of gradient refocused echo-planar fMRI susceptibility artifacts. NeuroImage, 6, 156-167.

Ollinger, J. M., Corbetta, M., \& Shulman, G. L. (2001a). Separating processes within a trial in event-related functional MRI - II. Analysis. Neuroimage, 13(1), 218-229. doi:10.1006/ Nimg.2000.0711

Ollinger, J. M., Shulman, G. L., \& Corbetta, M. (2001b). Separating processes within a trial in event-related functional MRI - I. The method. NeuroImage, 13(1), 210-217. doi:10.1006/Nimg.2000.0710

Otto, T., \& Poon, P. (2006). Dorsal hippocampal contributions to unimodal contextual conditioning. Journal of Neuroscience, 26 (24), 6603-6609. doi:10.1523/JNEUROSCI.1056-06.2006

Phillips, M. L., Drevets, W. C., Rauch, S. L., \& Lane, R. (2003). Neurobiology of emotion perception II: Implications for major psychiatric disorders. Biological Psychiatry, 54(5), 515-528.

Price, J. L., \& Drevets, W. C. (2010). Neurocircuitry of mood disorders. Neuropsychopharmacology, 35(1), 192-216. doi:10.1038/ Npp.2009.104

Rajkowska, G., \& Goldman-Rakic, P. S. (1995a). Cytoarchitectonid definition of prefrontal areas in normal human cortex: I. Remapping of areas 9 and 46 using quantitative criteria. Cerebral Cortex, $5,307-322$.

Rajkowska, G., \& Goldman-Rakic, P. S. (1995b). Cytoarchitectonic definition of prefrontal areas in the normal human cortex: II. Variability in locations of areas 9 and 46 and relationship to the talairach coordinate system. Cerebral Cortex, 5(4), 323-337.

Rao, U., Chen, L. A., Bidesi, A. S., Shad, M. U., Thomas, M. A., \& Hammen, C. L. (2010). Hippocampal changes associated with early-life adversity and vulnerability to depression. Biological Psychiatry, 67(4), 357-364. doi:10.1016/j.biopsych.2009.10.017

Robb, R. A. (2001). The biomedical imaging resource at Mayo Clinic. IEEE Transactions on Medical Imaging, 20(9), 854-867. doi: $10.1109 / 42.952724$

Roberson-Nay, R., McClure, E. B., Monk, C. S., Nelson, E. E., Guyer, A. E., Fromm, S. J., \& Pine, D. S. (2006). Increased amygdala activity during successful memory encoding in adolescent major depressive disorder: An FMRI study. Biological Psychiatry, 60 (9), 966-973. doi:10.1016/j.biopsych.2006.02.018

Rosso, I. M., Cintron, C. M., Steingard, R. J., Renshaw, P. F., Young, A. D., \& Yurgelun-Todd, D. A. (2005). Amygdala and hippocampus volumes in pediatric major depression. Biological Psychiatry, 57(1), 21-26. doi:10.1016/j.biopsych.2004.10.027

Sapolsky, R. M. (2000a). Glucocorticoids and hippocampal atrophy in neuropsychiatric disorders. Archives of General Psychiatry, 57 (10), 925-935.

Sapolsky, R. M. (2000b). The possibility of neurotoxicity in the hippocampus in major depression: A primer on neuron death. Biological Psychiatry, 48(8), 755-765

Savine, A. C., \& Braver, T. S. (2010). Motivated cognitive control: Reward incentives modulate preparatory neural activity during task-switching. Journal of Neuroscience, 30(31), 10294-10305. doi:10.1523/Jneurosci.2052-10.2010

Savitz, J., \& Drevets, W. C. (2009). Bipolar and major depressive disorder: Neuroimaging the developmental-degenerative divide. Neuroscience and Biobehavioral Reviews, 33(5), 699-771. doi:10.1016/j.neubiorev.2009.01.004

Sheline, Y. I., Barch, D. M., Donnelly, J. M., Ollinger, J. M., Snyder, A. Z., \& Mintun, M. A. (2001). Increased amygdala response to masked emotional faces in depressed subjects resolves with antidepressant treatment: An fMRI study. Biological Psychiatry, 50 (9), 651-658.

Sheline, Y. I., Barch, D. M., Price, J. L., Rundle, M. M., Vaishnavi, S. N., Snyder, A. Z., Raichle, M. E. (2009). The default mode network and self-referential processes in depression. Proc Natl Acad Sci U S A.

Sheline, Y. I., Gado, M. H., \& Kraemer, H. C. (2003). Untreated depression and hippocampal volume loss. The American Journal of Psychiatry, 160(8), 1516-1518.

Sheline, Y. I., Sanghavi, M., Mintun, M. A., \& Gado, M. H. (1999). Depression duration but not age predicts hippocampal volume loss in medically healthy women with recurrent major depression. Journal of Neuroscience, 19(12), 50345043.

Sheline, Y. I., Wang, P. W., Gado, M. H., Csernansky, J. G., \& Vannier, M. W. (1996). Hippocampal atrophy in recurrent major depression. Proceedings of the National Academy of Sciences of the United States of America, 93(9), 3908-3913.

Siegle, G. J., Steinhauer, S. R., Thase, M. E., Stenger, V. A., \& Carter, C. S. (2002). Can't shake that feeling: Event-related fMRI assessment of sustained amygdala activity in response to emotional information in depressed individuals. Biological Psychiatry, 51 (9), 693-707.

Siegle, G. J., Thompson, W., Carter, C. S., Steinhauer, S. R., \& Thase, M. E. (2007). Increased amygdala and decreased dorsolateral prefrontal BOLD responses in unipolar depression: Related and independent features. Biological Psychiatry, 61(2), 198-209. doi:10.1016/j.biopsych.2006.05.048

Small, S. A., Schobel, S. A., Buxton, R. B., Witter, M. P., \& Barnes, C. A. (2011). A pathophysiological framework of hippocampal dysfunction in ageing and disease. Nature Reviews Neuroscience, 12 (10), 585-601. doi:10.1038/nrn3085

Steffens, D. C., Byrum, C. E., McQuoid, D. R., Greenberg, D. L., Payne, M. E., Blitchington, T. F., \& Krishnan, K. R. (2000). Hippocampal volume in geriatric depression. Biological Psychiatry, 48(4), 301-309.

Steffens, D. C., McQuoid, D. R., Payne, M. E., \& Potter, G. G. (2011). Change in hippocampal volume on magnetic resonance imaging and cognitive decline among older depressed and nondepressed subjects in the neurocognitive outcomes of depression in the elderly study. The American Journal of Geriatric Psychiatry, 19 (1), 4-12. doi:10.1097/JGP.0b013e3181d6c245

Surguladze, S., Brammer, M. J., Keedwell, P., Giampietro, V., Young, A. W., Travis, M. J., \& Phillips, M. L. (2005). A differential pattern of neural response toward sad versus happy facial expressions in major depressive disorder. Biological Psychiatry, 57(3), 201-209. doi:10.1016/j.biopsych.2004.10.028

Talairach, J., \& Tournoux, P. (1988). Co-planar stereotaxic atlas of the human brain. New York: Thieme.

Thomas, K. M., Drevets, W. C., Dahl, R. E., Ryan, N. D., Birmaher, B., Eccard, C. H., \& Casey, B. J. (2001). Amygdala response to fearful faces in anxious and depressed children. Archives of General Psychiatry, 58(11), 1057-1063.

Tottenham, N., \& Sheridan, M. A. (2009). A review of adversity, the amygdala and the hippocampus: A consideration of developmental timing. Frontiers in Human Neuroscience, 3, 68. doi:10.3389/ neuro.09.068.2009

Tottenham, N., Tanaka, J. W., Leon, A. C., McCarry, T., Nurse, M., Hare, T. A., \& Nelson, C. (2009). The NimStim set of facial expressions: Judgments from untrained research participants. Psychiatry Research, 168(3), 242-249. doi:10.1016/ j.psychres.2008.05.006

Vakili, K., Pillay, S. S., Lafer, B., Fava, M., Renshaw, P. F., Bonello-Cintron, C. M., \& Yurgelun-Todd, D. A. (2000). Hippocampal volume in primary unipolar major depression: A 
magnetic resonance imaging study. Biological Psychiatry, 47 (12), 1087-1090.

Videbech, P., \& Ravnkilde, B. (2004). Hippocampal volume and depression: A meta-analysis of MRI studies. The American Journal of Psychiatry, 161(11), 1957-1966. doi:10.1176/appi.ajp.161.11.1957

Wang, L., Mamah, D., Harms, M. P., Karnik, M., Price, J. L., Gado, M. H., \& Csernansky, J. G. (2008). Progressive deformation of deep brain nuclei and hippocampal-amygdala formation in schizophrenia. Biological Psychiatry, 64(12), 1060-1068. doi:10.1016/ j.biopsych.2008.08.007

Ward, B. D., (2000). Simultaneous inference for FMRI data. Retrieved September 12, 2012, from http://afni.nimh.nih.gov/afni/doc/manual/ AlphaSim
Whalen, P. J., Shin, L. M., McInerney, S. C., Fischer, H., Wright, C. I., \& Rauch, S. L. (2001). A functional MRI study of human amygdala responses to facial expressions of fear versus anger. Emotion, $1(1), 70-83$.

Woon, F. L., Sood, S., \& Hedges, D. W. (2010). Hippocampal volume deficits associated with exposure to psychological trauma and posttraumatic stress disorder in adults: A meta-analysis. Progress in Neuro-Psychopharmacology \& Biological Psychiatry, 34(7), 1181-1188. doi:10.1016/j.pnpbp.2010.06.016

Yoon, T., Graham, L. K., \& Kim, J. J. (2011). Hippocampal lesion effects on occasion setting by contextual and discrete stimuli. Neurobiology of Learning and Memory, 95(2), 176-184. doi:10.1016/j.nlm.2010.07.001 